Araştırma Makalesi - Research Article

\title{
İki SUR Model Altında Ön Tahmin Edicilerin Kovaryans Matrisleri Üzerine Bazı Notlar
}

Görünürde ilişkisiz regresyon (SUR) modelleri denklemler arasında hataların ilişkili olduğu çoklu regresyon denklemlerinin ele alındığı lineer regresyon modellerinin uzantılarıdır. Bu çalışmada, SUR modelleri altında ön tahmin problemi ele alınmıştır. İki SUR modeli altında tüm bilinmeyen vektörlerin en iyi lineer yansız ön tahmin edicilerinin (BLUP' larının) istatistiksel özellikleri üzerine çeşitli sonuçlar verilmiştir. Özellikle, matrislerin bazı rank formülleri kullanılarak iki model altında BLUP'ların kovaryans matrisleri üzerine bazı sonuçlar elde edilmiştir.

1*Sorumlu yazar iletişim: nesring@sakarya.edu.tr (https://orcid.org/0000-0003-3233-5377)

Department of Statistics, Sakarya University, Sakarya, 54187, Turkey

2İletişim: yuce.nevin@gmail.com (https://orcid.org/0000-0002-2967-2930)

Department of Mathematics, Sakarya University, Sakarya, 54187, Turkey 


\title{
Some Notes on Covariance Matrices of Predictors under two SUR Models
}

\begin{abstract}
Seemingly unrelated regression (SUR) models are extensions of linear regression models by considering multiple regression equations with correlated errors among equations. In this study, prediction problem under SUR models are considered. Several results are given on statistical properties of the best linear unbiased predictors (BLUPs) of all unknown vectors under two SUR models. Especially, some results established on covariance matrices of BLUPs under two models by using some rank formulas of matrices.
\end{abstract}




\section{INTRODUCTION}

Several number of models with individual relations with each other can encounter in some statistical problems. These models can have correlated error terms among each other although they look like unrelated if the same data or an amount of same independent variables are used for the models. For example, we can consider the problem of investment on some companies at the same industry. Each company can be affected by similar cases under the same periods. In this case, it can be expected that error terms of set of models may be simultaneously correlated. Seemingly unrelated regression (SUR) models are consider multiple regression equations having correlation in error terms. They have attracted considerable interest in recent years; see e.g., [1-4], since these models were proposed by Zellner [5]. The method for joint estimation of unknown parameters by combining the equations was given by [5-7]. For the related topic; see, e.g., [8-13].

Consider a pair of SUR models formulated by

$$
\begin{aligned}
& \mathcal{M}_{1}: y_{1}=X_{1} \beta_{1}+\varepsilon_{1}, E\left(\varepsilon_{1}\right)=0, D\left(\varepsilon_{1}\right)=\sigma_{11} I_{n}:=\Sigma_{11}, \\
& \mathcal{M}_{2}: y_{2}=X_{2} \beta_{2}+\varepsilon_{2}, E\left(\varepsilon_{2}\right)=0, D\left(\varepsilon_{2}\right)=\sigma_{22} I_{n}:=\Sigma_{22},
\end{aligned}
$$

which are based on the equation

$$
y_{i j}=x_{i j 1} \beta_{i 1}+\cdots+x_{i j p_{i}} \beta_{i p_{i}}+\varepsilon_{i j}
$$

where $y_{i}=\left(y_{i j}\right) \in \mathbb{R}^{n \times 1}$ is an observable random vector, $X_{i}=\left(x_{i j t}\right) \in \mathbb{R}^{n \times p_{i}}$ is a known matrix of arbitrary rank, $\beta_{i}=\left(\beta_{i j}\right) \in \mathbb{R}^{p_{i} \times 1}$ is an unknown parameter vector, and $\varepsilon_{i}=\left(\varepsilon_{i j}\right) \in \mathbb{R}^{n \times 1}$ is an error vector with $\operatorname{cov}\left(\varepsilon_{i}, \varepsilon_{k}\right)=\sigma_{i k} I_{n}:=\Sigma_{i k}$ for $i, k=1,2, j=1,2, \ldots, n$, and $t=1, \ldots, p_{i}$.

In order to establish some results on predictions/estimations of all unknown parameters under models $\mathcal{M}_{1}$ and $\mathcal{M}_{2}$ we can consider the following general linear function

$$
\phi_{i}=K_{i} \beta_{i}+H_{i} \varepsilon_{i}
$$

for given matrices $K_{i} \in \mathbb{R}^{k \times p_{i}}$ and $H_{i} \in \mathbb{R}^{k \times n}, i=1,2$. According to the assumptions in (1) and (2),

$$
E\left(\phi_{i}\right)=K_{i} \beta_{i}, D\left(\phi_{i}\right)=H_{i} \Sigma_{i i} H_{i}^{\prime} \text {, and } \operatorname{cov}\left(\phi_{i}, y_{i}\right)=H_{i} \Sigma_{i i},
$$

$i=1,2$. The parameter vector $\phi_{i}$ in (4) is said to be predictable under $\mathcal{M}_{i}$ if there exists a linear statistic $L_{i} y_{i}$ with $L_{i} \in \mathbb{R}^{k \times n}$ such that $E\left(L_{i} y_{i}-\phi_{i}\right)=0$ holds; see [14], this is equivalently written as

$$
\mathcal{C}\left(K_{i}^{\prime}\right) \subseteq \mathcal{C}\left(X_{i}^{\prime}\right)
$$

$i=1$,2. (6) also corresponds the estimability condition of $K_{i} \beta_{i}$ under $\mathcal{M}_{i}$; see [15]. Let $\phi_{i}$ be predictable under $\mathcal{M}_{i}$. If there exists $L_{i} y_{i}$ such that

$$
D\left(L_{i} y_{i}-\phi_{i}\right)=\min \text { subject to } E\left(L_{i} y_{i}-\phi_{i}\right)=0
$$

holds in the Löwner partial ordering, the linear statistic $L_{i} y_{i}$ is defined to be the best linear unbiased predictor (BLUP) of $\phi_{i}$, a term introduced by Goldberger [16], and is denoted by $L_{i} y_{i}=\mathrm{BLUP}_{\mathcal{M}_{i}}\left(\phi_{i}\right)=$ $\operatorname{BLUP}_{\mathcal{M}_{i}}\left(K_{i} \beta_{i}+H_{i} \varepsilon_{i}\right), i=1,2$. If $H_{i}=0$, BLUP of $\phi_{i}$ reduces to the best linear unbiased estimator (BLUE) of $K_{i} \beta_{i}$ under $\mathcal{M}_{i}$, expressed as $L_{i} y_{i}=\operatorname{BLUE}_{\mathcal{M}_{i}}\left(\phi_{i}\right)=\operatorname{BLUE}_{\mathcal{M}_{i}}\left(K_{i} \beta_{i}\right)$.

Prediction problems under SUR models are one of the main subjects in the statistical inference of the models. The best known predictors/estimators of unknown vectors in the models are the BLUPs/BLUEs. In this 
study, we consider two SUR models and give some results on covariance matrices between BLUPs of all unknown vectors under these models in theoretical point of view. Characterization of statistical properties of BLUPs under considered models involves some complex matrix expressions. Therefore, we use some matrix rank formulas for simplifying heavy matrix operations while establishing results.

Throughout this paper, the symbol $\mathbb{R}^{m \times n}$ stands for the set of all $m \times n$ real matrices. For $A \in \mathbb{R}^{m \times n}$, the notations $A^{\prime}, r(A)$, and $\mathcal{C}(A)$, denote the transpose, the rank, and the column space of $A$, respectively. $I_{m}$ denotes the identity matrix of order $m$. The Moore-Penrose generalized inverse of $A$ denoted by $A^{+}$, is defined to be the unique solution $G$ satisfying the four matrix equation $A G A=A, G A G=G,(A G)^{\prime}=A G,(G A)^{\prime}=G A$. Furthermore, $P_{A}=A A^{+}, E_{A}=A^{\perp}=I_{m}-A A^{+}$, and $F_{A}=I_{n}-A^{+} A$ stand for the orthogonal projectors.

\section{FORMULAS FOR BLUPS UNDER TWO SUR MODELS}

In this section, firstly we give some rank formulas of matrices. Then we give fundamental BLUP equation of $\phi_{i}$ and related properties of BLUPs. In what follows, it is assumed that the model $\mathcal{M}_{i}$ is consistent, i.e., $y_{i} \in$ $\mathcal{C}\left[X_{i} \quad \Sigma_{i i}\right]$ with probability $1, i=1,2 ;$ see [17].

The well-known formulas for rank of matrices collected in the following lemma; see [18].

Lemma 1 Let $A \in \mathbb{R}^{m \times n}, B \in \mathbb{R}^{m \times k}, C \in \mathbb{R}^{l \times n}$, and $D \in \mathbb{R}^{l \times k}$. Then

(a) $r\left[\begin{array}{ll}A & B\end{array}\right]=r(A)+r\left(E_{A} B\right)=r(B)+r\left(E_{B} A\right)$,

(b) $r\left[\begin{array}{l}A \\ C\end{array}\right]=r(A)+r\left(C F_{A}\right)=r(C)+r\left(A F_{C}\right)$,

(c) If $\mathcal{C}(B) \subseteq \mathcal{C}(A)$ and $\mathcal{C}\left(C^{\prime}\right) \subseteq \mathcal{C}\left(A^{\prime}\right)$, then $r\left[\begin{array}{ll}A & B \\ C & D\end{array}\right]=r(A)+r\left(D-C A^{+} B\right)$.

The following lemma on BLUP for $\phi_{i}$ under model $\mathcal{M}_{i}$ is derived from Theorem 3.2 in [19].

Lemma 2 Let $\phi_{i}$ be predictable under $\mathcal{M}_{i}$. Then

$$
L_{i} y_{i}=\operatorname{BLUP}_{\mathcal{M}_{i}}\left(\phi_{i}\right) \Leftrightarrow L_{i}\left[\begin{array}{ll}
X_{i} & \Sigma_{i i} X_{i}^{\perp}
\end{array}\right]=\left[\begin{array}{ll}
K_{i} & H_{i} \sum_{i i} X_{i}{ }^{\perp}
\end{array}\right]
$$

General solution of this equation and corresponding BLUP of $\phi_{i}$ is

$$
L_{i} y_{i}=\operatorname{BLUP}_{\mathcal{M}_{i}}\left(\phi_{i}\right)=\left(\left[\begin{array}{ll}
K_{i} & H_{i}{\Sigma_{i i}}_{X_{i}}{ }^{\perp}
\end{array}\right]\left[\begin{array}{ll}
X_{i} & \Sigma_{i i} X_{i}{ }^{\perp}
\end{array}\right]^{+}+U_{i}\left[\begin{array}{ll}
X_{i} & \Sigma_{i i} X_{i}{ }^{\perp}
\end{array}\right]^{\perp}\right) y_{i}
$$

where $U_{i} \in \mathbb{R}^{k \times n}$ is arbitrary, $i=1,2$. Further, $\operatorname{BLUP}_{\mathcal{M}_{i}}\left(\phi_{i}\right)$ satisfies the following statements

$$
\begin{aligned}
& D\left[\operatorname{BLUP}_{\mathcal{M}_{i}}\left(\phi_{i}\right)\right]=\left[\begin{array}{lll}
K_{i} & H_{i} \Sigma_{i i} X_{i}{ }^{\perp}
\end{array}\right]\left[\begin{array}{ll}
X_{i} & \Sigma_{i i} X_{i}{ }^{\perp}
\end{array}\right]^{+} \sum_{i i}\left(\left[\begin{array}{ll}
K_{i} & H_{i} \Sigma_{i i} X_{i}{ }^{\perp}
\end{array}\right]\left[\begin{array}{ll}
X_{i} & \Sigma_{i i} X_{i}{ }^{\perp}
\end{array}\right]^{+}\right)^{\prime}, \\
& \operatorname{cov}\left[\operatorname{BLUP}_{\mathcal{M}_{i}}\left(\phi_{i}\right), \phi_{i}\right]=\left[\begin{array}{ll}
K_{i} & H_{i} \Sigma_{i i} X_{i}{ }^{\perp}
\end{array}\right]\left[\begin{array}{ll}
X_{i} & \Sigma_{i i} X_{i}{ }^{\perp}
\end{array}\right]^{+} \sum_{i i} H_{i}{ }^{\prime}, \\
& D\left[\phi_{i}-\operatorname{BLUP}_{\mathcal{M}_{i}}\left(\phi_{i}\right)\right]=\left(\left[\begin{array}{ll}
K_{i} & H_{i} \Sigma_{i i} X_{i}{ }^{\perp}
\end{array}\right] W_{i}^{+}-H_{i}\right) \Sigma_{i i}\left(\left[\begin{array}{ll}
K_{i} & H_{i} \Sigma_{i i} X_{i}{ }^{\perp}
\end{array}\right] W_{i}^{+}-H_{i}\right)^{\prime},
\end{aligned}
$$

where $\mathrm{W}_{i}=\left[\begin{array}{ll}X_{i} & \Sigma_{i i} X_{i}{ }^{\perp}\end{array}\right], i=1,2$. Furthermore, the following statements hold.

(a) $r\left[X_{i} \quad \Sigma_{i i} X_{i}{ }^{\perp}\right]=r\left[\begin{array}{ll}X_{i} & \Sigma_{i i}\end{array}\right], \mathcal{C}\left[X_{i} \quad \Sigma_{i i} X_{i}{ }^{\perp}\right]=\mathcal{C}\left[\begin{array}{ll}X_{i} & \Sigma_{i i}\end{array}\right]$, and $\mathcal{C}\left(X_{i}\right) \cap \mathcal{C}\left(\sum_{i i} X_{i}{ }^{\perp}\right)=\{0\}$,

(b) $\operatorname{BLUP}_{\mathcal{M}_{i}}\left(\phi_{i}\right)$ is unique with probability $1 \Leftrightarrow y_{i} \in \mathcal{C}\left[\begin{array}{ll}X_{i} & \Sigma_{i i}\end{array}\right]$ with probability 1 , 
(c) $L_{i}$ is unique $\Leftrightarrow r\left[\begin{array}{ll}X_{i} & \Sigma_{i i}\end{array}\right]=n, i=1,2$.

We note that BLUP of $\phi_{i}$ and ordinary least square predictor (OLSP) of $\phi_{i}$ coincide since $\Sigma_{i i}=\sigma_{i i} I_{n}$, $i=1,2$. Then the results in Lemma 2 reduce the following results, for details, see; [18].

$$
\begin{aligned}
& L_{i} y_{i}=\operatorname{BLUP}_{\mathcal{M}_{i}}\left(\phi_{i}\right)=\left(K_{i} X_{i}{ }^{+}+H_{i} X_{i}{ }^{\perp}\right) y_{i}, \\
& D\left[\operatorname{BLUP}_{\mathcal{M}_{i}}\left(\phi_{i}\right)\right]=\sigma_{i i}\left(K_{i} X_{i}{ }^{+}+H_{i} X_{i}{ }^{\perp}\right)\left(K_{i} X_{i}^{+}+H_{i} X_{i}{ }^{\perp}\right)^{\prime}, \\
& \operatorname{cov}\left[\operatorname{BLUP}_{\mathcal{M}_{i}}\left(\phi_{i}\right), \phi_{i}\right]=\sigma_{i i}\left(K_{i} X_{i}^{+}+H_{i} X_{i}{ }^{\perp}\right) H_{i}{ }^{\prime}, \\
& D\left[\phi_{i}-\operatorname{BLUP}_{\mathcal{M}_{i}}\left(\phi_{i}\right)\right]=\sigma_{i i}\left(K_{i} X_{i}^{+}-H_{i} P_{X_{i}}\right)\left(K_{i} X_{i}{ }^{+}-H_{i} P_{X_{i}}\right)^{\prime} .
\end{aligned}
$$

\section{COVARIANCE MATRIX BETWEEN BLUPS UNDER TWO SUR MODELS}

In this section, by presenting general approach to SUR models, we give the main result on the covariance matrices between BLUPs of unknown vectors under models $\mathcal{M}_{1}$ and $\mathcal{M}_{2}$. Then, we give some consequences which correspond special cases.

Theorem 1 Assume that $\phi_{1}$ and $\phi_{2}$ is predictable under models $\mathcal{M}_{1}$ and $\mathcal{M}_{2}$, respectively, i.e., (6) holds. Let denote

$$
M=\left[\begin{array}{ccccc}
\Sigma_{12} & \Sigma_{11} & X_{1} & 0 & 0 \\
\Sigma_{22} & 0 & 0 & X_{2} & 0 \\
X_{2}{ }^{\prime} & 0 & 0 & 0 & K_{2}^{\prime}-X_{2}^{\prime} H_{2}^{\prime} \\
0 & X_{1}{ }^{\prime} & 0 & 0 & 0 \\
0 & 0 & K_{1}-H_{1} X_{1} & 0 & 0
\end{array}\right]
$$

Then

$$
\begin{aligned}
& r\left(\operatorname{cov}\left\{\phi_{1}-\operatorname{BLUP}_{\mathcal{M}_{1}}\left(\phi_{1}\right), \phi_{2}-\operatorname{BLUP}_{\mathcal{M}_{2}}\left(\phi_{2}\right)\right\}\right)=r(M)-r\left[\begin{array}{ll}
X_{1} & \Sigma_{11}
\end{array}\right]- \\
& -r\left[\begin{array}{ll}
X_{2} & \Sigma_{22}
\end{array}\right]-r\left(X_{1}\right)-r\left(X_{2}\right) \text {. }
\end{aligned}
$$

Furthermore, $\phi_{1}-\operatorname{BLUP}_{\mathcal{M}_{1}}\left(\phi_{1}\right)$ and $\phi_{2}-\operatorname{BLUP}_{\mathcal{M}_{2}}\left(\phi_{2}\right)$ are uncorrelated if and only if

$$
r(M)=r\left[\begin{array}{ll}
X_{1} & \Sigma_{11}
\end{array}\right]+r\left[\begin{array}{ll}
X_{2} & \Sigma_{22}
\end{array}\right]+r\left(X_{1}\right)+r\left(X_{2}\right) .
$$

Proof. From (10) - (12), we can write

$$
\begin{aligned}
& r\left(\operatorname{cov}\left\{\phi_{1}-\operatorname{BLUP}_{\mathcal{M}_{1}}\left(\phi_{1}\right), \phi_{2}-\operatorname{BLUP}_{\mathcal{M}_{2}}\left(\phi_{2}\right)\right\}\right) \\
& \quad=r\left(\left(\left[\begin{array}{ll}
K_{1} & H_{1} \Sigma_{11} X_{1}{ }^{\perp}
\end{array}\right] W_{1}^{+}-H_{1}\right) \Sigma_{12}\left(\left[\begin{array}{ll}
K_{2} & H_{2} \Sigma_{22} X_{2}{ }^{\perp}
\end{array}\right] W_{2}{ }^{+}-H_{2}\right)^{\prime}\right),
\end{aligned}
$$

where $\mathrm{W}_{i}=\left[\begin{array}{ll}X_{i} & \sum_{i i} X_{i}^{\perp}\end{array}\right], i=1,2$. We can apply Lemma 1(c) to (20). Then we obtain

$$
r\left(\operatorname{cov}\left\{\phi_{1}-\operatorname{BLUP}_{\mathcal{M}_{1}}\left(\phi_{1}\right), \phi_{2}-\operatorname{BLUP}_{\mathcal{M}_{2}}\left(\phi_{2}\right)\right\}\right)
$$




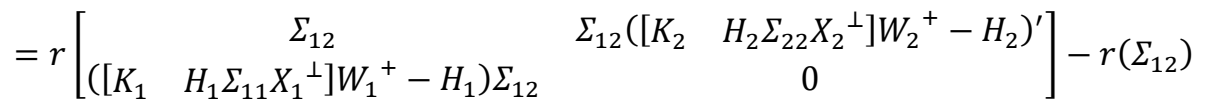

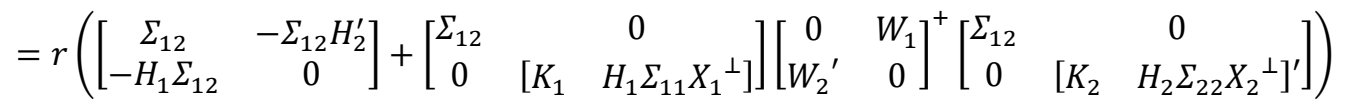

$$
\begin{aligned}
& -r\left(\Sigma_{12}\right) .
\end{aligned}
$$

We can reapply Lemma 1 (c) to (21) since $\mathcal{C}\left(\left[\begin{array}{ll}K_{i} & H_{i} \Sigma_{i i} X_{i}^{\perp}\end{array}\right]^{\prime}\right) \subseteq \mathcal{C}\left(W_{i}^{\prime}\right)$ and $\mathcal{C}\left(\Sigma_{i j}\right) \subseteq \mathcal{C}\left(W_{i}\right)$ hold. Then by using Lemma 1 and simplifying elementary block matrix operations, we obtain

$$
\begin{aligned}
& r\left(\operatorname{cov}\left\{\phi_{1}-\operatorname{BLUP}_{\mathcal{M}_{1}}\left(\phi_{1}\right), \phi_{2}-\operatorname{BLUP}_{\mathcal{M}_{2}}\left(\phi_{2}\right)\right\}\right) \\
& =r\left[\begin{array}{ccccc}
0 & -X_{1} & -\Sigma_{11} X_{1}{ }^{\perp} & \Sigma_{12} & 0 \\
-X_{2}^{\prime} & 0 & 0 & 0 & K_{2}^{\prime} \\
-X_{2}{ }^{\perp} \Sigma_{22} & 0 & 0 & 0 & X_{2}{ }^{\perp} \Sigma_{22} H_{2}^{\prime} \\
\Sigma_{12} & 0 & 0 & \Sigma_{12} & -\Sigma_{12} H_{2}^{\prime} \\
0 & K_{1} & H_{1} \Sigma_{11} X_{1}{ }^{\perp} & -H_{1} \Sigma_{12} & 0
\end{array}\right]-r\left[\begin{array}{cc}
0 & {\left[\begin{array}{ll}
X_{1} & \Sigma_{11} X_{1}{ }^{\perp}
\end{array}\right]} \\
{\left[X_{2}\right.} & \Sigma_{22} X_{2}{ }^{\perp}
\end{array}\right]^{\prime} \quad r\left(\Sigma_{12}\right) \\
& =r\left[\begin{array}{cccc}
-\Sigma_{12} & -X_{1} & -\Sigma_{11} X_{1}{ }^{\perp} & \Sigma_{12} H_{2}^{\prime} \\
-X_{2}^{\prime} & 0 & 0 & K_{2}^{\prime} \\
-X_{2}{ }^{\perp} \Sigma_{22} & 0 & 0 & X_{2}{ }^{\perp} \Sigma_{22} H_{2}^{\prime} \\
H_{1} \Sigma_{12} & K_{1} & H_{1} \Sigma_{11} X_{1}{ }^{\perp} & -H_{1} \Sigma_{12} H_{2}^{\prime}
\end{array}\right]-r\left[\begin{array}{lll}
X_{1} & \Sigma_{11} X_{1}{ }^{\perp}
\end{array}\right]-r\left[\begin{array}{ll}
X_{2} & \Sigma_{22} X_{2}{ }^{\perp}
\end{array}\right] \\
& =r\left[\begin{array}{ccccc}
\Sigma_{12} & X_{1} & \Sigma_{11} & 0 & 0 \\
X_{2}^{\prime} & 0 & 0 & K_{2}^{\prime}-X_{2}^{\prime} H_{2}^{\prime} & 0 \\
\Sigma_{22} & 0 & 0 & 0 & X_{2} \\
0 & K_{1}-H_{1} X_{1} & 0 & 0 & 0 \\
0 & 0 & X_{1}^{\prime} & 0 & 0
\end{array}\right]-r\left[\begin{array}{lll}
X_{1} & \Sigma_{11}
\end{array}\right]-r\left[\begin{array}{ll}
X_{2} & \Sigma_{22}
\end{array}\right]-r\left(X_{1}\right)-r\left(X_{2}\right) \\
& =r\left[\begin{array}{ccccc}
\Sigma_{12} & \Sigma_{11} & X_{1} & 0 & 0 \\
\Sigma_{22} & 0 & 0 & X_{2} & 0 \\
X_{2}{ }^{\prime} & 0 & 0 & 0 & K_{2}^{\prime}-X_{2}^{\prime} H_{2}^{\prime} \\
0 & X_{1}^{\prime} & 0 & 0 & 0 \\
0 & 0 & K_{1}-H_{1} X_{1} & 0 & 0
\end{array}\right]-r\left[\begin{array}{ll}
X_{1} & \Sigma_{11}
\end{array}\right]-r\left[\begin{array}{ll}
X_{2} & \Sigma_{22}
\end{array}\right]-r\left(X_{1}\right)-r\left(X_{2}\right) .
\end{aligned}
$$

From (22), we obtained the required results in (18) and (19).

The following result is an immediate consequences of Theorem 1.

Corollary 1 Assume that $K_{1} \beta_{1}$ and $K_{2} \beta_{2}$ are estimable under models $\mathcal{M}_{1}$ and $\mathcal{M}_{2}$, respectively, i.e., (6) holds. Then

$$
r\left(\operatorname{cov}\left\{\operatorname{BLUE}_{\mathcal{M}_{1}}\left(K_{1} \beta_{1}\right), \operatorname{BLUE}_{\mathcal{M}_{2}}\left(K_{2} \beta_{2}\right)\right\}\right)
$$




$$
=r\left[\begin{array}{ccccc}
\Sigma_{12} & \Sigma_{11} & X_{1} & 0 & 0 \\
\Sigma_{22} & 0 & 0 & X_{2} & 0 \\
X_{2}^{\prime} & 0 & 0 & 0 & K_{2}^{\prime} \\
0 & X_{1}^{\prime} & 0 & 0 & 0 \\
0 & 0 & K_{1} & 0 & 0
\end{array}\right]-r\left[\begin{array}{ll}
X_{1} & \Sigma_{11}
\end{array}\right]-r\left[\begin{array}{ll}
X_{2} & \Sigma_{22}
\end{array}\right]-r\left(X_{1}\right)-r\left(X_{2}\right) .
$$

Furthermore, $\operatorname{BLUE}_{\mathcal{M}_{1}}\left(K_{1} \beta_{1}\right)$ and $\operatorname{BLUE}_{\mathcal{M}_{2}}\left(K_{2} \beta_{2}\right)$ are uncorrelated if and only if

$$
r\left[\begin{array}{ccccc}
\Sigma_{12} & \Sigma_{11} & X_{1} & 0 & 0 \\
\Sigma_{22} & 0 & 0 & X_{2} & 0 \\
X_{2}^{\prime} & 0 & 0 & 0 & K_{2}^{\prime} \\
0 & X_{1}{ }^{\prime} & 0 & 0 & 0 \\
0 & 0 & K_{1} & 0 & 0
\end{array}\right]=r\left[\begin{array}{lll}
X_{1} & \Sigma_{11}
\end{array}\right]+r\left[\begin{array}{ll}
X_{2} & \Sigma_{22}
\end{array}\right]+r\left(X_{1}\right)+r\left(X_{2}\right) .
$$

$X_{1} \beta_{1}$ and $X_{2} \beta_{2}$ are always estimable under models $\mathcal{M}_{1}$ and $\mathcal{M}_{2}$, respectively. Then

$$
\begin{aligned}
r\left(\operatorname{cov}\left\{\operatorname{BLUE}_{\mathcal{M}_{1}}\left(X_{1} \beta_{1}\right), \operatorname{BLUE}_{\mathcal{M}_{2}}\left(X_{2} \beta_{2}\right)\right\}\right)= & r\left[\begin{array}{ccc}
\Sigma_{12} & \Sigma_{11} & 0 \\
\Sigma_{22} & 0 & X_{2} \\
0 & X_{1}^{\prime} & 0
\end{array}\right] \\
& -r\left[\begin{array}{lll}
X_{1} & \Sigma_{11}
\end{array}\right]-r\left[\begin{array}{ll}
X_{2} & \Sigma_{22}
\end{array}\right] .
\end{aligned}
$$

In particular, the following statements are equivalent.

(a) $\operatorname{BLUE}_{\mathcal{M}_{1}}\left(X_{1} \beta_{1}\right)$ and $\operatorname{BLUE}_{\mathcal{M}_{2}}\left(X_{2} \beta_{2}\right)$ are uncorrelated.

(b) $\varepsilon_{1}-\operatorname{BLUP}_{\mathcal{M}_{1}}\left(\varepsilon_{1}\right)$ and $\varepsilon_{2}-\operatorname{BLUP}_{\mathcal{M}_{2}}\left(\varepsilon_{2}\right)$ are uncorrelated.

(c) $r\left[\begin{array}{ccc}\Sigma_{12} & \Sigma_{11} & 0 \\ \Sigma_{22} & 0 & X_{2} \\ 0 & X_{1}^{\prime} & 0\end{array}\right]=r\left[\begin{array}{ll}X_{1} & \Sigma_{11}\end{array}\right]+r\left[\begin{array}{ll}X_{2} & \Sigma_{22}\end{array}\right]$.

We represent the general approach to SUR models in Theorem 1 and Corollary 1 . The results in (18), (23), and (25) can also be expressed as follows since $\Sigma_{i k}=\sigma_{i k} I_{n}, i, k=1,2$.

$$
\begin{aligned}
& r\left(\operatorname{cov}\left\{\phi_{1}-\mathrm{BLUP}_{\mathcal{M}_{1}}\left(\phi_{1}\right), \phi_{2}-\mathrm{BLUP}_{\mathcal{M}_{2}}\left(\phi_{2}\right)\right\}\right) \\
& =r\left[\begin{array}{ccc}
\sigma_{12} X_{1}^{\prime} X_{2} & X_{1}^{\prime} X_{1} & 0 \\
X_{2}^{\prime} X_{2} & 0 & K_{2}^{\prime}-X_{2}^{\prime} H_{2}^{\prime} \\
0 & K_{1}-H_{1} X_{1} & 0
\end{array}\right]-r\left(X_{1}\right)-r\left(X_{2}\right) . \\
& r\left(\operatorname{cov}\left\{\operatorname{BLUE}_{\mathcal{M}_{1}}\left(K_{1} \beta_{1}\right), \mathrm{BLUE}_{\mathcal{M}_{2}}\left(K_{2} \beta_{2}\right)\right\}\right)=r\left[\begin{array}{ccc}
\sigma_{12} X_{1}^{\prime} X_{2} & X_{1}^{\prime} X_{1} & 0 \\
X_{2}^{\prime} X_{2} & 0 & K_{2}^{\prime} \\
0 & K_{1} & 0
\end{array}\right]-r\left(X_{1}\right)-r\left(X_{2}\right) . \\
& r\left(\operatorname{cov}\left\{\operatorname{BLUE}_{\mathcal{M}_{1}}\left(X_{1} \beta_{1}\right), \mathrm{BLUE}_{\mathcal{M}_{2}}\left(X_{2} \beta_{2}\right)\right\}\right)=r\left(X_{1}^{\prime} X_{2}\right) .
\end{aligned}
$$




\section{CONCLUSION}

In this study, we present a general approach to SUR models by giving some statistical properties of BLUPs under two SUR models by using some rank formulas of matrices. In order to establish general results on predictor/estimator, we consider general linear function of all unknown vectors under models. Especially, we establish some results on covariance matrices between BLUPs of unknown vectors under two SUR models by addressing the subject theoretically. Although some results related to the predictors under general linear regression models can be applied to SUR models, algebraic properties of BLUPs under SUR models need to be clearly expressed and the formulations of statistical properties are worth to be considered to obtain more statistical inference of the models.

\section{REFERENCES}

[1] Gong, L. (2019). Establishing equalities of OLSEs and BLUEs under seemingly unrelated regression Models. Journal of Statistical Theory and Practice, 13:5.

[2] Hou, J., Zhao, Y. (2019). Some remarks on a pair of seemingly unrelated regression models. Open Math., 17, 979-989.

[3] Jiang, H., Qian, J., Sun, Y. (2020). Best linear unbiased predictors and estimators under a pair of constrained seemingly unrelated regression models. Stat. Probab. Lett., 158, 108669.

[4] Sun, Y., Ke, R., Tian, Y. (2014). Some overall properties of seemingly unrelated regression models. $A d v$. Stat. Anal., 98 (2), 103-120.

[5] Zellner, A. (1962). An efficient method of estimating seemingly unrelated regressions and tests for aggregation bias. J. Am. Stat. Assoc., 57, 348-368.

[6] Zellner, A. (1963). Estimators for seemingly unrelated regression equations: some exact finite sample results. J. Am. Stat. Assoc., 58, 977-992.

[7] Zellner, A., Huang, D. S. (1962). Further properties of efficient estimators for seemingly unrelated regression equations. Int. Econ. Rev., 3, 300-313.

[8] Baksalary, J. K., Kala, R. (1979). On the prediction problem in the seemingly unrelated regression equations model. Statistics, 10, 203-208.

[9] Baksalary, J. K., Trenkler, G. (1989). The efficiency of OLS in a seemingly unrelated regressions model. Econ. Theory, 5, 463-465.

[10] Dwivedi, T. D., Srivastava, V. K. (1978). Optimality of least squares in the seemingly unrelated regression model. J. Econ., 7, 391-395.

[11] Foschi, P., Kontoghiorghes, E. J. (2002). Seemingly unrelated regression model with unequal size observations: computational aspects. Comput. Stat. Data Anal., 41, 211-229.

[12] Liu, A. Y. (2002). Efficient estimation of two seemingly unrelated regression equations. Journal of Multivariate Analysis, 82, 445-456.

[13] Srivastava, V. K., Giles, D. E. A. (1987). Seemingly Unrelated Regression Equations Model. Marcel Dekker, New York.

[14] Tian, Y. (2015). A new derivation of BLUPs under random-effects model. Metrika, 78, 905-918.

[15] Alalouf, I. S., Styan, G. P. H. (1979). Characterizations of estimability in the general linear model. Ann. Stat., 7, 194-200. 
[16] Goldberger, A. S. (1962). Best linear unbiased prediction in the generalized linear regression models. J. Amer. Stat. Assoc., 57, 369-375.

[17] Rao, C. R. (1973). Representations of best linear unbiased estimators in the Gauss-Markoff model with a singular dispersion matrix. J. Multivariate Anal., 3, 276-292.

[18] Marsaglia, G., Styan, G. P. H. (1974). Equalities and inequalities for ranks of matrices. Linear Multilinear Algebra, 2, 269-292.

[19] Tian, Y. (2017). Matrix rank and inertia formulas in the analysis of general linear models. Open Math., 15, 126-150. 